En conclusion, le séquençage à haut débit représente une révolution dans la recherche, avec l'identification de la diversité du génome humain, la dissection des bases moléculaires des maladies mendéliennes ou complexes. On assiste également à une révolution médicale, du fait de la rapidité du diagnostic, de la diminution de l'errance diagnostique et de l'accès plus rapide à une prise en charge précoce, un suivi adapté, un traitement médical personnalisé et une prise en charge adaptée des familles.

L'avenir de la génomique est le développement et l'accès au séquençage du génome pour les patients. Pour cela, il nous faut mieux comprendre les variations codantes, les variations non codantes, les interactions entre les gènes et avec l'environnement, afin de permettre une médecine de précision basée sur l'interprétation des variations génomiques individuelles. $\diamond$

State of play of knowledge and of technologies in the field of genomics

\section{LIENS D'INTÉRÊT}

L'auteur déclare n'avoir aucun lien d'intérêt concernant les données publiées dans cet article.

\title{
Le plan France Médecine Génomique 2025 et les maladies rares
}
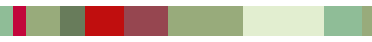

> Nous vivons une révolution en médecine grâce à la génomique, que I'on compare souvent à la révolution de la radiographie ou de l'IRM. Les outils à disposition sont, en effet, extraordinaires.

À partir de 2010, de nombreuses initiatives ont été lancées au niveau international, autour de la médecine personnalisée centrée sur les analyses génétiques, en matière de recherche et de soins. On peut notamment citer l'Initiative Genomics England, la Precision Medecine Initiative aux États-Unis et le remboursement du NGS en Estonie.

En avril 2015, le Premier ministre a chargé Yves Lévy d'étudier le problème de la médecine génomique en France et de faire des propositions. S'en est suivi un travail d'octobre 2015 à mars 2016, avec des contributions inclusives de nombreux professionnels, de beaucoup d'administrations, d'institutions, d'agences, d'industriels, d'associations de patients. Plus de 160 personnalités ont été mobilisées autour de la préparation du plan France Médecine Génomique.

Cette préparation a comporté une analyse de la situation française par rapport à la situation internationale, l'innovation et les enjeux industriels, un état des lieux en France et des propositions portant sur les infrastructures et les organisations.

Des propositions ont été formulées pour permettre à tous les patients de bénéficier de l'apport de la génomique dans le diagnostic, pour le pronostic et les soins de leur maladie, qui constituent le plan France Médecine génomique, remis en juin 2016 au Premier ministre, qui au-delà des changements gouvernementaux soutient concrètement le plan. Se sont alors mis en place les premiers axes et travaux du plan.
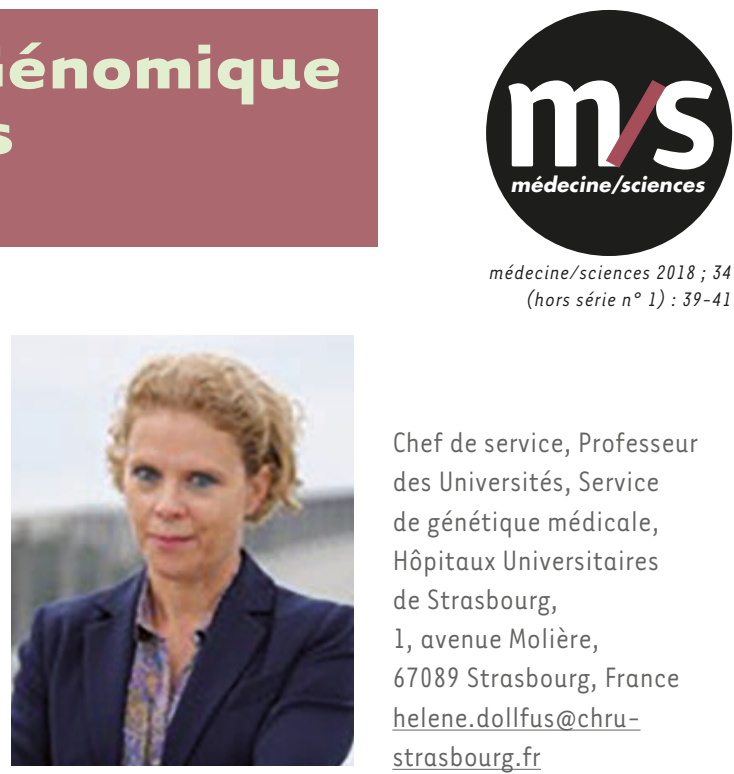

(hors série $n^{\circ}$ 1) : 39-41

Chef de service, Professeur des Universités, Service de génétique médicale, Hôpitaux Universitaires de Strasbourg, 1 , avenue Molière, 67089 Strasbourg, France helene.dollfus@chrustrasbourg.fr

\section{Les étapes de la mise en œuvre}

Le plan vise à intégrer le séquençage du génome en routine dans la pratique clinique, et à développer une filière nationale de médecine génomique en partenariat avec la recherche et les industriels (Figure 1). La France veut en effet redevenir leader dans ce domaine.

Le plan est organisé autour de trois objectifs et 14 mesures. II s'adresse à toutes les maladies. Certains objectifs sont génériques et seront utilisables dans le domaine du cancer comme celui des maladies rares.

Tout d'abord, il a été décidé, pour structurer le séquençage haut débit, de mettre en place des plateformes au niveau du territoire dans les prochaines années. Ces plateformes sont supposées couvrir l'ensemble des pathologies susceptibles d'avoir besoin de séquençage haut débit. En décembre 2016, un appel à projets a été lancé à l'attention des plateformes. Deux plateformes de séquençage ont été sélectionnées: SEDOiA 


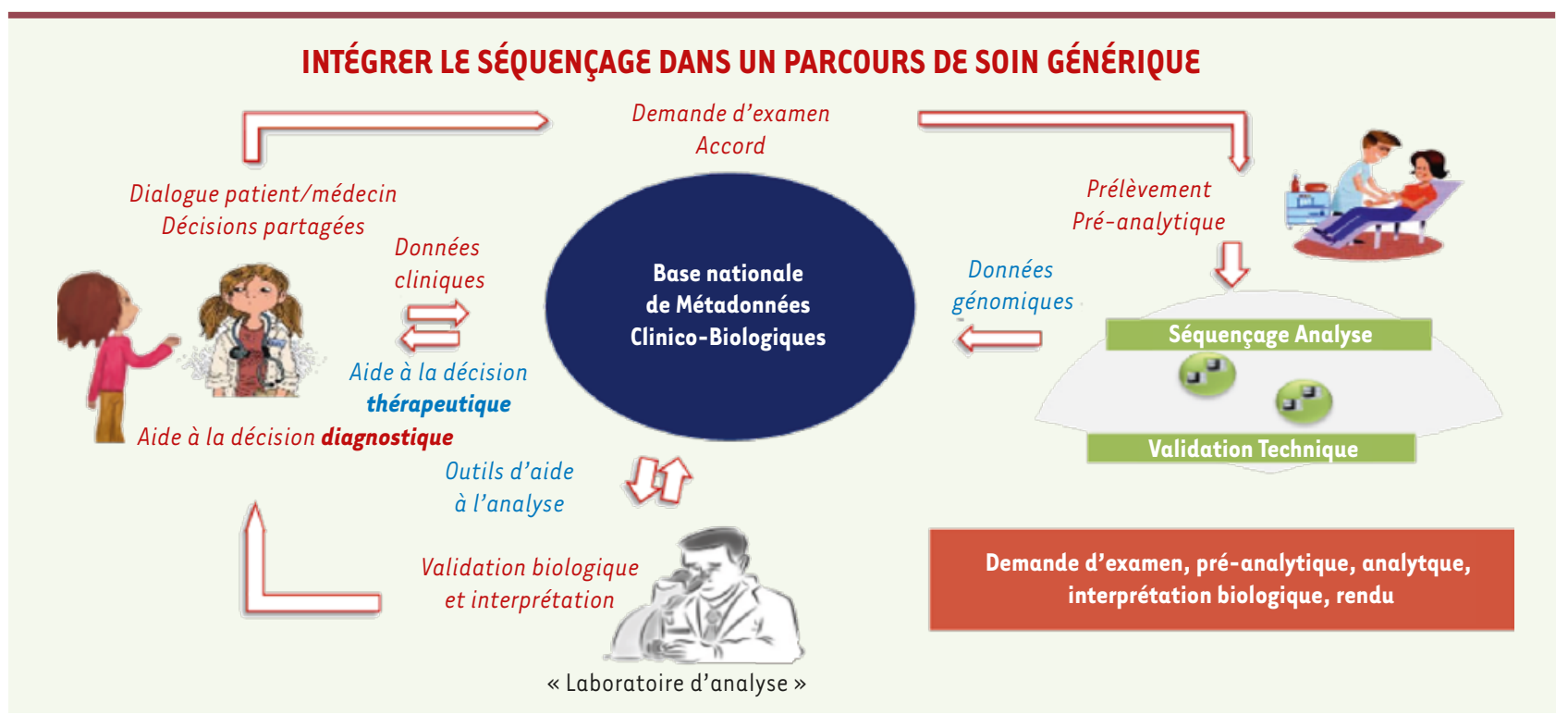

Figure 1. Intégrer le séquençage du génome dans la pratique clinique.

et AURAGEN. Sera également créé un collecteur analyseur de données, afin de traiter et d'exploiter les données. Une autre structure commune permettra de suivre l'innovation technologique et les progrès autour du séquençage. II s'agira d'un centre de référence en matière d'innovation et d'expertise de transfert.

Une mesure consiste à évaluer les indications et à déterminer ce qui se passe sur le terrain lors de la mise en œuvre des différentes mesures du plan, notamment en termes de prise en charge financière par l'assurance maladie et se trouve actuellement traitée avec la HAS.

Une mesure porte sur les nouveaux métiers dont il faudra disposer sur le terrain clinique, comme celui de conseiller génétique spécialisé en génomique.

L'évaluation médico-économique de l'ensemble du dispositif et sa position au niveau international constitue une autre priorité.

Avant toute chose, ce plan vise à améliorer le parcours de soins et a été conçu autour de ce dernier. Le prélèvement sera acheminé vers une plateforme de séquençage. Les séquences seront alignées, annotées, voire comparées dans certaines circonstances. Les laboratoires hospitaliers valideront et interpréteront avec le clinicien, dans la perspective d'un rendu au patient.

Je m'étendrai davantage sur la mesure $n^{\circ} 5$, qui a comme objectif de lever les verrous technologiques, cliniques et réglementaires sur le parcours de soin. Dès septembre 2016 , les quatre projets pilotes ont été lancés, portant respectivement sur la variabilité de la population générale, le diabète, le cancer et les maladies rares.

Le projet DEFIDIAG est le projet Maladies rares du plan France Médecine Génomique, qui porte sur la déficience intellectuelle. L'Inserm est promoteur de ce projet pilote comme des trois autres et à ce titre le pôle de recherche clinique de l'Inserm est très impliqué dans l'élaboration de ce projet pilote, qui a vocation à être un démonstrateur de l'efficacité du plan tel qu'il été conçu.

Nous avons beaucoup de chance, car notre comité de pilotage est extrêmement actif. Des groupes de travail ont été mis en place. Le groupe «méthodologie, data et évaluation médico-économique » est porté avec brio par les différentes équipes du CHU et de l'université de Dijon. D'autres groupes travaillent sur le circuit du patient, les laboratoires et la bio-informatique, le budget, l'éthique et le réglementaire.

\section{Le séquençage pour le diagnostic des déficiences intellectuelles}

Le choix de la déficience intellectuelle s'est imposé de lui-même, car il s'agit de la première cause de consultation dans les services de génétique. $1 \%$ à $3 \%$ de la population générale souffrirait d'un déficit intellectuel, soit environ un million de Français. C'est une cause majeure d'errance diagnostique et un exemple important d'hétérogénéité clinique et moléculaire. Des centaines de gènes sont impliqués. II est très important pour les patients de connaître la cause moléculaire afin d'adapter la prise en charge et d'obtenir un conseil génétique.

L'objectif principal est de comparer les causes génétiques identifiées pour les patients avec déficience intellectuelle, en utilisant le séquençage du génome complet, en l'analysant de manière séquentielle et en le comparant à la stratégie nationale de référence.

Les objectifs secondaires visent par exemple à détecter les anomalies de structure, identifier d'autres diagnostics et identifier potentiellement de nouveaux gènes transférés à la recherche. Grâce aux efforts des équipes de Dijon, une partie portera sur l'efficience, c'est-àdire le coût et l'efficacité de la nouvelle stratégie.

Une étude d'impact dirigée par l'équipe de Dijon au niveau économique, médical et psychosocial permettra 
d'estimer le coût de l'errance diagnostique potentiellement évitable en utilisant cette technologie et l'impact de la technologie par rapport à la prise en charge des patients.

Une étude qualitative portera sur la perception des parents et patients par rapport à cette analyse.

L'étude reposera sur un seul lieu de séquençage, à savoir le Centre National de Recherche en Génomique Humaine (CNRGH). 1277 patients seront recrutés avec leurs parents, portant le nombre de participants à environ 3800 . Les patients seront des patients vus en consultation, des patients pris à partir de l'âge de deux ans, ainsi que des adultes, quel que soit le degré de gravité de leur déficience. L'étude sera multicentrique; six laboratoires experts et douze centres cliniques recruteurs participeront. Un aspect de faisabilité sera également étudié.

Il est important de voir comment tout ceci s'intégrera dans le parcours de soin du patient.

Les retombées attendues sont multiples. Pour les patients, il s'agit de connaître les causes de la maladie. Pour les professionnels, il s'agit de mettre en situation et de mieux structurer les discussions entre médecins et laboratoires. Les données générées alimenteront une base qui pourra être utilisée sous conditions par les scientifiques dans le cadre de programmes ultérieurs pour poursuivre l'avancée des connaissances. Les coûts et durées seront estimés, afin d'aboutir à une tarification, une prise en charge et un remboursement en lien avec la mesure ad hoc du plan.

Le comité scientifique d'évaluation devrait se tenir en 2018, pour une première inclusion de malades mi-2018. Pour conclure, le Plan France Médecine Génomique est élaboré depuis trente mois. Deux plateformes ont été désignées. Les quatre projets pilotes ont été lancés et la dynamique est excellente. II s'agit maintenant d'assurer une jonction avec le Plan national Maladies rares $n^{\circ} 3$. Un axe de ce plan est en effet dédié au diagnostic. Il faut que nous parvenions à répondre aux impératifs internationaux, selon lesquels chaque patient devrait recevoir un diagnostic avec les moyens dont disposent la médecine et la science à ce moment-là. $\diamond$ France Genomics Medicine 2025 plan

\section{LIENS D'INTÉRÊT}

L'auteur déclare n'avoir aucun lien d'intérêt concernant les données publiées dans cet article.

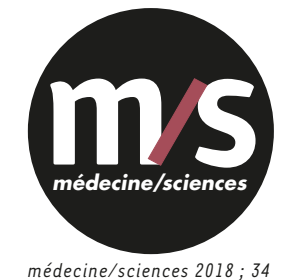

(hors série $n^{\circ} 1$ ) : $41-42$

\section{La génomique en diagnostic}

de routine

Benoît Arveiler

$>$ En France, les laboratoires de diagnostic des maladies rares et d'oncogénétique constitutionnelle localisés dans les Centre Hospitaliers Universitaires et les Centre de Lutte contre le Cancer sont organisés en réseau depuis une quinzaine d'années sous l'égide de l'Association Nationale des Praticiens de Génétique Moléculaire (ANPGM). Chaque laboratoire est en charge du diagnostic de certaines maladies ou groupes de maladies, parfois de façon unique au niveau national. Ceci garantit une forte expertise et constitue un gage de qualité pour le diagnostic. L'ensemble des laboratoires s'est doté depuis environ cinq ans des technologies de séquençage à haut débit, permettant grâce à l'analyse de panels de gènes d'atteindre un niveau d'exhaustivité jusque-là inatteignable et de réduire considérablement les délais de rendu des résultats.

L'évolution vers le séquençage de l'exome ou du génome entier est indispensable pour les maladies génétiquement très hétérogènes, lorsque le séquençage de panels n'est pas satisfaisant ou irréalisable. C'est l'un des objectifs du Plan France Médecine Génomique 2025 (PFMG 2025).

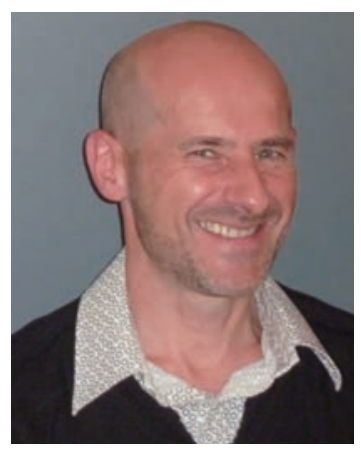

Professeur des universitésPraticien Hospitalier, Responsable du Laboratoire de génétique moléculaire, $\mathrm{CHU}$ de Bordeaux-GH Pellegrin, Place Amélie Raba-Léon, 33076 Bordeaux Cedex, France. benoit.arveiler@chubordeaux.fr

Une articulation devra être trouvée entre les plateformes de séquençage à très haut débit mises en place dans le cadre du PFMG 2025 et le réseau des laboratoires de génétique pour garantir le circuit des prélèvements, le partage des séquences produites, leur interprétation et la validation bioinformatique et fonctionnelle par les biologistes. Une validation finale sera réalisée avec les cliniciens prescripteurs au sein de réunions 